\title{
A unique case of rapidly progressive diffuse pulmonary nodules
}

\author{
Madina Weiler ${ }^{1}$, Angelica Puopolo ${ }^{1}$, William Newmarch ${ }^{1 *}$ and Brian Casserly ${ }^{2}$ \\ ${ }^{1}$ Graduate Entry Medical School, University of Limerick, Limerick, Ireland \\ ${ }^{2}$ Respiratory Division, University Hospital Limerick, Limerick, Ireland
}

\begin{abstract}
The following case report describes a unique presentation of primary malignant fibrous histiocytoma (MFH) originating in the right ventricle. A 71-year-old male presented to the emergency department with tachycardia, dyspnea, tachypnea, and left leg pitting edema, seven weeks following a right total hip arthroplasty. Six weeks prior to presentation, the patient was diagnosed with pulmonary embolism and five pulmonary nodules based on computed tomography (CT) angiography. Laboratory studies at this time indicated anemia of chronic disease, consistent with results from six weeks earlier. Broncho-alveolar lavage (BAL) cytology suggested adenocarcinoma. CT angiography demonstrated a dramatic increase in the amount and extent of the pulmonary nodules previously noted in addition to an unresolved embolus of the right upper lobe. CT-guided biopsy was performed and confirmed a definitive diagnosis of non-small cell lung cancer. Two days after CT-guided biopsy, the patient developed acute hypotension and hypoxic respiratory failure rapidly progressing to asystole and subsequent death. Autopsy revealed a primary intra-cavitary malignant fibrous histiocytoma in the right ventricle, and multiple nodular tumours throughout the lung parenchyma with chest wall invasion. These findings allowed for a revision of the diagnosis and clinical-pathological picture. This case illustrates the limitations of radiological imaging in visualizing vascular structures, and the continued importance of autopsy in achieving a complete and correct pathological diagnosis. Moreover, this case draws light to the unclear diagnostic criteria for MFH and lack of effective treatment options. The longest documented survival of MFH is eight years, which was achieved with orthotopic cardiac transplantation.
\end{abstract}

\section{Introduction}

Primary malignant cardiac neoplasms are an extremely rare occurrence, as the vast majority of primary cardiac neoplasms are benign and typically of the atrial myxoma type [1]. Primary malignant cardiac neoplasms account for approximately $25 \%$ of all primary cardiac tumours, and $75 \%$ of malignant cardiac tumors are sarcomas, including angiosarcoma, malignant fibrous histiocytoma (MFH) and rhabdomyosarcoma [1]. MFH frequently arises in left atrium, like myxomas, but can be identified histologically by the presence of histiocytes and malignant giant cells arranged in a characteristic storiform pattern [2]. As with most cardiac tumours, prognosis of $\mathrm{MFH}$ is relatively poor due to the increased likelihood of metastasis and local recurrence. The following case report outlines an incidence of primary cardiac MFH diagnosed in a 71-year-old man. Additionally, it highlights the importance of autopsy as an invaluable tool in the diagnosis of MFH as it is a rare tumour with indistinct histologic criteria.

\section{Case report}

A 71-year-old male presented to the emergency department with increasing dyspnea and left leg swelling following a right total hip arthroplasty one week prior. On further questioning the patient reported a 100 pack-year smoking history and marginal asbestos exposure. Vital signs were normal on presentation and the only laboratory finding of significance was a decreased hemoglobin level of $10.3 \mathrm{~g} / \mathrm{dL}$, consistent with anemia of chronic disease. Initial imaging of the chest revealed mild cardiomegaly with no infiltrates, masses or effusions on plain film (Figure 1). Further imaging with computed tomography (CT) angiography demonstrated a pulmonary embolism of the right upper lobe and five pulmonary nodules not previously visible on chest radiograph (Figure 2). Echocardiogram was unremarkable except for an increased systolic pulmonary artery pressure of $50 \mathrm{mmHg}$. Warfarin therapy was commenced with a follow-up CT arranged for one month's time.

Six weeks following the initial presentation, the patient returned to the emergency department with similar complaints of increasing dyspnea on exertion and left leg pain and swelling. He had not been on warfarin for two days prior to this admission as his prescription had expired. On examination, the patient was found to be febrile, tachycardic and tachypnic, requiring $2 \mathrm{~L}$ of oxygen to maintain a saturation of $97 \%$. Auscultation of his chest revealed decreased bibasilar breath sounds. Both cardiac and abdominal examinations were unremarkable. Left lower limb 2+ pitting edema was noted with extension upwards to the mid-thigh. No cyanosis or finger clubbing was present. Repeat blood investigations and imaging studies were performed and compared to the results obtained 6 weeks prior.

Like the reported blood results on the initial presentation, hemoglobin levels were low $(10.4 \mathrm{~g} / \mathrm{dL})$. Both the erythrocytesedimentation rate and C-reactive protein were elevated at $69 \mathrm{mg} / \mathrm{L}$ and

Correspondence to: William Newmarch, Graduate Entry Medical School, University of Limerick, Limerick, Ireland Tel: +353 (83) 187-9766; E-mail: 14076926@studentmail.ul.ie

Key words: Malignant fibrous histiocytoma, primary cardiac tumors, tumor emboli, lung nodules

Received: January 25, 2017; Accepted: March 01, 2017; Published: March 04 2017 


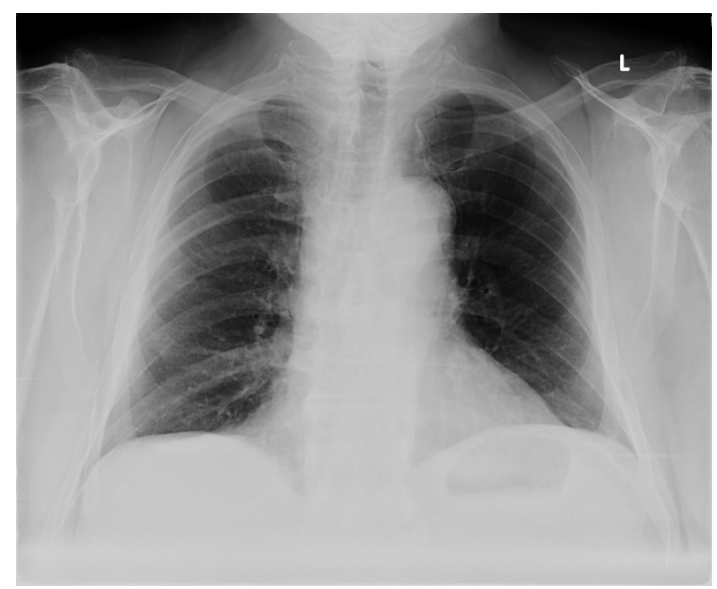

Figure 1. Chest radiograph demonstrating mild cardiomegaly without masses or infiltrates.

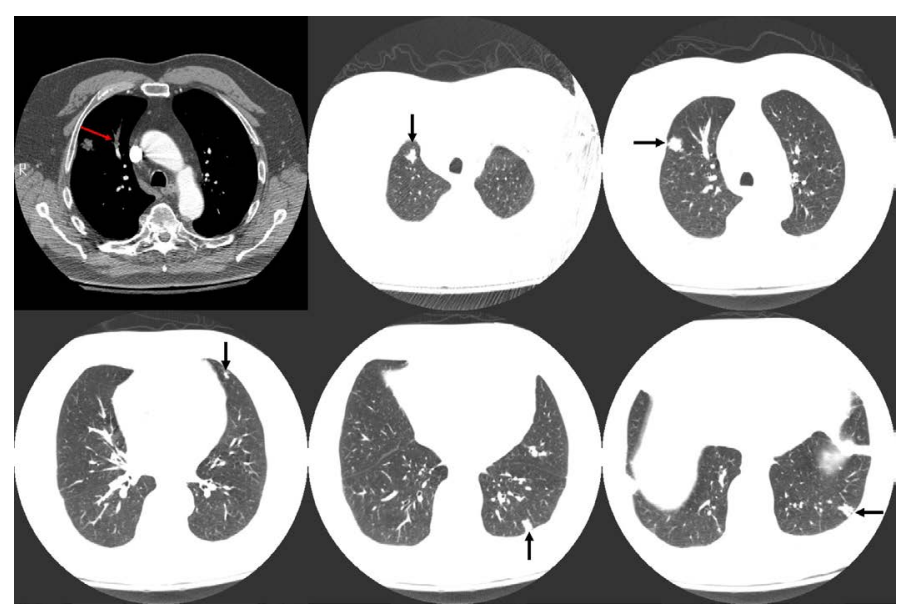

Figure 2. Computed tomography (CT) imaging of the chest revealing a right upper lobe (RUL) embolism (red arrow) in addition to five pulmonary nodules (black arrows).

$56 \mathrm{mg} / \mathrm{L}$, respectively. Additional laboratory investigations including full blood count (FBC) and differential, platelets, chemistries, renal function, urinalysis, liver function panel, international normalized ratio (INR), anti-nuclear antibody (ANA), rheumatoid factor (RF), anti-nuclear cytoplasmic antibody (ANCA), anti-glomerular basement membrane, blood cultures, urine cultures and sputum cultures were all normal.

Imaging studies revealed diffuse pulmonary nodules and bilateral effusions on chest radiograph (Figure 3). CT angiography demonstrated a dramatic increase in the amount and extent of the pulmonary nodules previously noted in addition to an unresolved embolus of the right upper lobe (Figure 4).

\section{Pathology}

Additional tests performed to establish a diagnosis included bronchoscopy and broncho-alveolar lavage (BAL). Bronchoscopy revealed scant secretions and an absence of endobronchial lesions. BAL cytology suggested adenocarcinoma, while BAL cultures were negative, and CT-guided biopsy was performed to assist in confirming a definitive diagnosis. Two days following the biopsy procedure, the patient's condition deteriorated as he developed acute hypotension and hypoxic respiratory failure rapidly progressing to asystole. This necessitated urgent intubation and resuscitation. Pathological examination of the biopsy confirmed a diagnosis of non-small cell lung cancer. Given the news of this diagnosis, the family made the decision to withdraw the patient's life support.

Autopsy of the lungs uncovered multiple nodular tumours throughout the lung parenchyma with chest wall invasion (Figure 5) in addition to several smaller nodules dispersed throughout the parietal pleura and mediastinum. Examination of the heart revealed a primary intra-cavitary tumour in the right ventricle which prompted a review of previous imaging studies. No other primary sites of tumor were identified. On closer examination of prior imaging investigations, the primary tumour can be visualised at the apex of the right ventricle (Figure 6). This revised clinic-pathologic picture, in combination with the biopsy specimens (Figure 7), led to the final diagnosis of primary cardiac malignant fibrous histiocytoma (MFH) with tumor emboli to the lung resulting in type 1 respiratory failure.

\section{Discussion}

Primary tumors of the heart are extremely rare, with an incidence of less than 0.1 percent on autopsy [3, 4]. Moreover, a large proportion of these tumors are benign. Conversely, metastatic disease involving the heart is 100 to 1,000 times more common [5]. Soft-tissue sarcomas are a rare group of malignant tumors of mesenchymal origin that

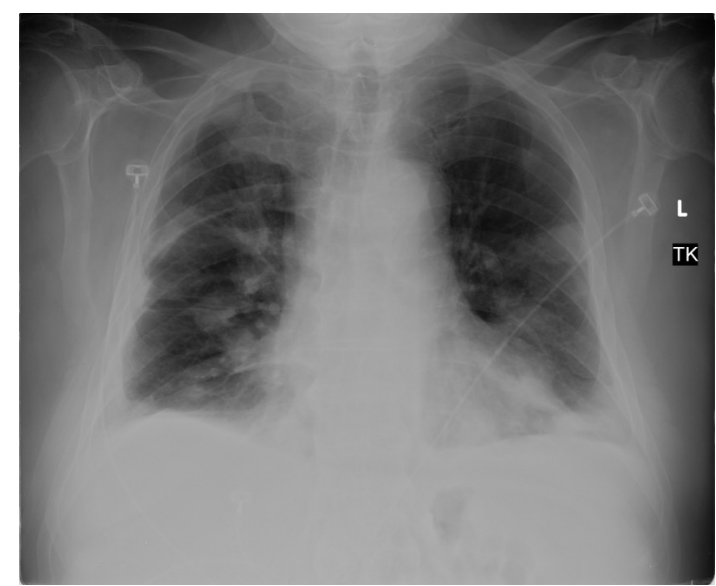

Figure 3. Repeat chest radiograph showing diffuse pulmonary nodules and bilateral pleural effusions.

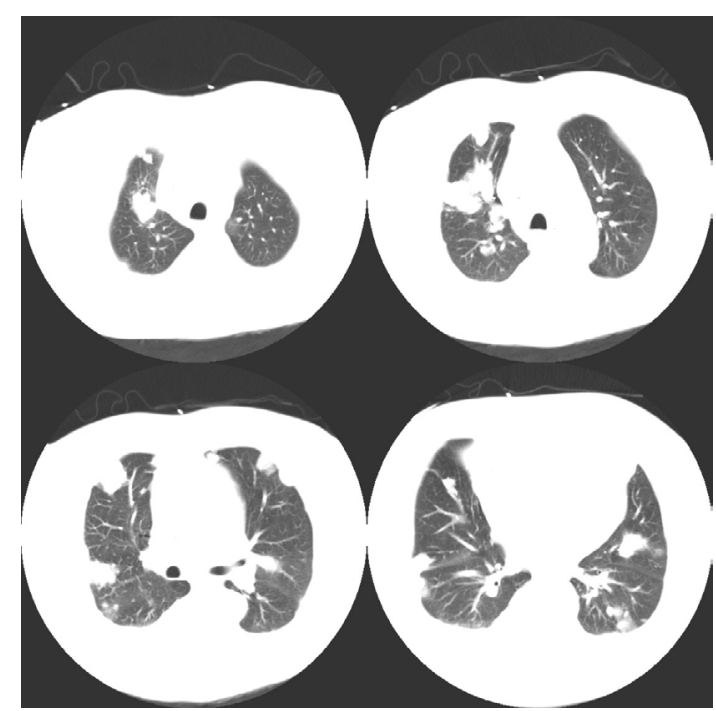

Figure 4. Chest $\mathrm{CT}$ revealing multiple nodules throughout both lung fields. 


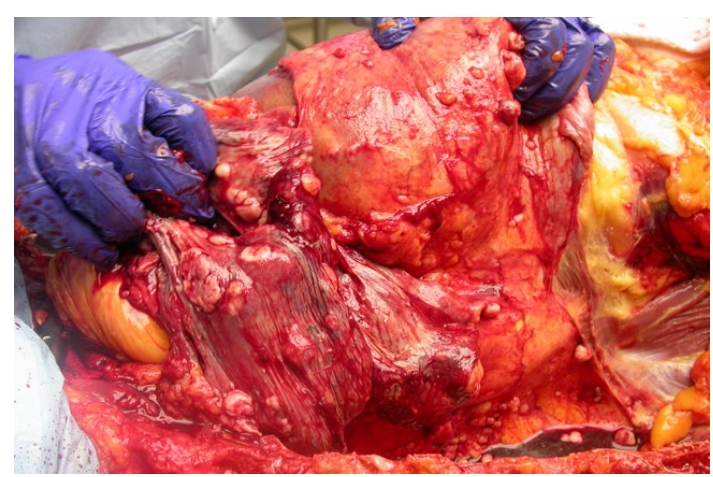

Figure 5. The CT-guided biopsy site as seen on autopsy. The tumor has invaded the chest wall.

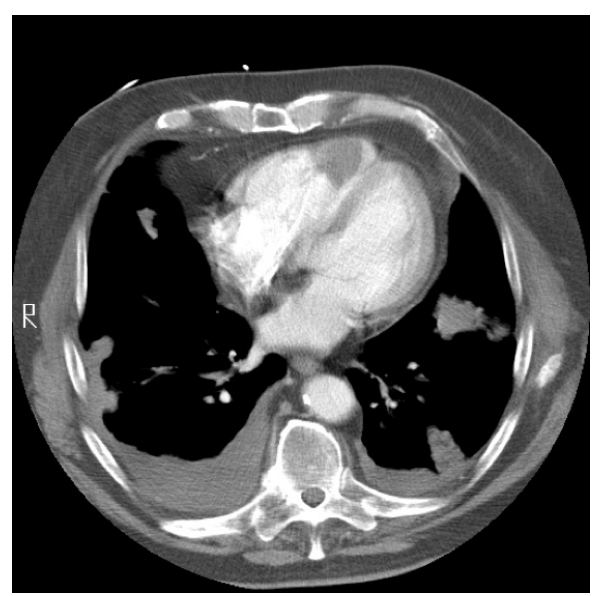

Figure 6. Chest CT highlighting the intra-cavitary tumor in the right ventricle.

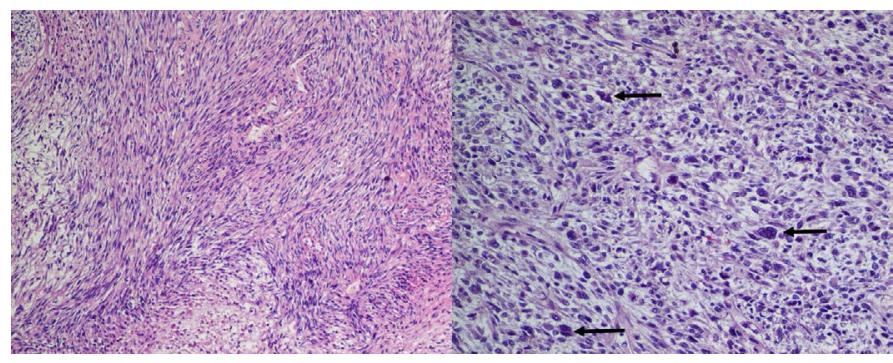

Figure 7. (A) Autopsy specimen of a representative lung mass (hematoxylin-eosin stain original magnification x100). (B) Pleomorphic multi-nucleated giant cells (black arrows) in a background of fibroblasts and histiocyte-like cells (hematoxylin-eosin stain; original magnification $\mathrm{x} 250$ )

account for less than $1 \%$ of all adult malignancies annually [6]. MFH, also termed "undifferentiated pleomorphic sarcoma" or "pleomorphic rhabdomyosarcoma", is one of the most common histologic subtypes in adults [7]. However, primary MFH of the heart is quite rare as it represents only $1.6 \%$ of all primary cardiac malignancies [8]. The first reported case of primary cardiac MFH was published in 1978, with a total of 91 cases mentioned in the literature to date [9]. This includes eight that originated in the right ventricle. In the reported cases, ages ranged from 14 to 80 years with more than half (54.9\%) involving the left atrium.

The presentation of cardiac MFH is typically a result of the tumor directly affecting cardiac function, leading to symptoms of dyspnea, arrhythmia, and syncope. In one review of 47 cases, dyspnea was the most common presenting complaint (74\%) followed by heart palpitations or chest discomfort (40\%) [10]. Our case is unique in that the local tumor in the right ventricle remained relatively small while the lung parenchyma was almost entirely invaded by tumor emboli. As a result, our patient developed severe hypoxemia without significant cardiac symptoms.

The treatment options for $\mathrm{MFH}$ are currently limited. The treatment plan depends on the tumor location, size, histological grade, and metastatic spread. Radiotherapy is generally not considered a viable therapeutic option as the effective doses are known to be cardiotoxic [11]. Chemotherapy has several limitations as well, most notably low response rates [7]. Furthermore, doxorubicin, an effective chemotherapeutic drug for the treatment of soft-tissue sarcomas, is cardiotoxic. Thus, its use and effectiveness in the treatment of cardiac sarcomas is unfortunately restricted [12]. Surgical resection with clear margins is the treatment of choice for localized tumors. In addition, surgical resection is necessary to ameliorate symptoms and to obtain a histologic diagnosis despite a high incidence of local recurrence and distant metastasis [10]. Due to the aggressive nature of this tumor, the average survival time is less than one year [13]. Orthotopic heart transplantation (OHT) has been reported, but the evidence of a clear benefit for MFH is limited [14]. However, one case described a local recurrence of $\mathrm{MFH}$ seven years after $\mathrm{OHT}$ for an unresectable right ventricular MFH [15].

The peripheral, multi-lobar pattern of nodules seen on CT imaging suggested hematogenous spread of the tumor. Differential diagnoses considered included pulmonary emboli with infarct, septic emboli, tumor emboli, vasculitis, atypical infection, or cryptogenic organizing pneumonia (COP). The absence of any infectious symptoms in addition to the presence of pulmonary embolism pointed toward malignancy. However, the rapidly progressive nature of the disease evolution is not typical for malignancy unless tumor embolization is considered.

The misdiagnosis on two separate histologic samples in this case highlights both the importance of autopsy in educating the medical community, and the challenges in making this diagnosis $[16,17]$. The diagnostic criteria for MFH is a continuous subject of debate amongst pathologists [13]. In fact, the "malignant fibrous histiocytoma" category was removed from the 2013 World Health Organization (WHO) Classification of Tumors of Soft Tissue and Bone and replaced with an "undifferentiated/unclassified sarcoma" category that reflects the lack of distinguishing histologic, immunohistochemical, or genetic features [18]. Despite this, MFH remains accepted as a distinct clinicopathologic entity. Typical histology, as in this case, reveals fibroblastlike spindle cells and histiocyte-like round or polygonal cells (Figure 7B) accompanied by pleomorphic multinucleated giant cells (Figure 7B, black arrows) in a "storiform" or "palisading" pattern (Figure 7A) consistent with the diagnosis of MFH. Pathologic diagnosis requires that there be no specific features of differentiation other than collagen production and phagocytosis [19].

\section{Clinical pearls}

This case illustrates the continued importance of autopsy, which revealed the initial pathological misdiagnosis and demonstrated how grossly under-appreciated the extent of the disease was, despite the sensitivity of CT scan.

For pulmonologists and chest radiologists, this case underscores how vascular structures often represent a "blind spot" on imaging and emphasizes the importance of carefully examining all structures seen on a chest CT. MFH is a rare primary tumor with poorly defined histologic criteria that can make definitive diagnosis quite challenging. While no 
effective treatment exists to date, orthotopic heart transplantation has resulted in the longest documented survival.

Although a unique case, tumor embolization from primary cardiac tumors should be included in the differential diagnosis of rapidly enlarging pulmonary parenchymal nodules in a pattern suggesting hematogenous spread.

\section{References}

1. Neragi-Miandoab S, Kim J, Vlahakes GJ (2007) Malignant Tumours of the Heart: A Review of Tumour Type, Diagnosis and Therapy. Clin Oncol(R Coll Radiol) 19: 748756. [Crossref]

2. Yaliniz H, Salih OK, Tokcan A (2008) Malignant Fibrous Histiocytoma of the Heart. Tex Heart Inst J 35: 84-85. [Crossref]

3. Reynen K (1996) Frequency of primary tumors of the heart. Am J Cardiol 77: 107. [Crossref]

4. Lam KY, Dickens P, Chan AC (1993) Tumors of the heart. A 20-year experience with a review of 12,485 consecutive autopsies. Arch Pathol Lab Med 117: 1027-1031. [Crossref]

5. Butany J, Nair V, Naseemuddin A, Nair GM, Catton C, et al. (2005) Cardiac tumours: diagnosis and management. Lancet Oncol 6: 219-228. [Crossref]

6. Siegel RL, Miller KD, Jemal A (2017) Cancer Statistics. CA Cancer J Clin 67: 7-30. [Crossref]

7. Milicic D, Juretic A, Bulum J, Saric N, Bisof V, et al. (2007) Primary malignant fibrous histiocytoma of the heart with skeletal muscles metastases. $J$ Card Surg 22: 513-516. [Crossref]

8. Sato M, Suenaga E, Senaha S, Furutachi A (2007) Primary malignant fibrous histiocytoma of the heart. Gen Thorac Cardiovasc Surg 55: 29-31. [Crossref]
9. Shah AA, Churg A, Sbarbaro JA, Sheppard JM, Lamberti J (1978) Malignant fibrous histiocytoma of the heart presenting as an atrial myxoma. Cancer 42: 2466-2471. [Crossref]

10. Okamoto K, Kato S, Katsuki S, Wada Y, Toyozumi Y, et al. (2001) Malignant fibrous histiocytoma of the heart: case report and review of 46 cases in the literature. Intern Med 40: 1222-1226. [Crossref]

11. Gaya AM, Ashford RF (2005) Cardiac complications of radiation therapy. Clin Oncol (R Coll Radiol) 17: 153-159. [Crossref]

12. Spira AI, Ettinger DS (2002) The use of chemotherapy in soft-tissue sarcomas. Oncologist 7: 348-359. [Crossref]

13. Schena S, Caniglia A, Agnino A, Caruso G, Ferlan G (2000) Survival following treatment of a cardiac malignant fibrous histiocytoma. Chest 118: 271-273. [Crossref]

14. Gowdamarajan A, Michler RE (2000) Therapy for primary cardiac tumors: is there a role for heart transplantation? Curr Opin Cardiol 15: 121-125. [Crossref]

15. Akhter SA, McGinty J, Konys JJ, Giesting RM, Merrill WH (2004) Recurrent primary cardiac malignant fibrous histiocytoma following orthotopic heart transplantation. $J$ Heart Lung Transplant 23: 1447-1450. [Crossref]

16. Burton JL, Underwood J (2007) Clinical, educational, and epidemiological value of autopsy. Lancet 369: 1471-1480. [Crossref]

17. Goldman L, Sayson R, Robbins S, Cohn LH, Bettmann M, et al. (1983) The value of the autopsy in three medical eras. N Engl J Med 308: 1000-1005. [Crossref]

18. Doyle LA (2014) Sarcoma classification: an update based on the 2013 World Health Organization Classification of Tumors of Soft Tissue and Bone. Cancer 120: 17631774. [Crossref]

19. Yves Glock, Binon JP, Rocchichioli JP, Christophe Duboucher, Rharid Kreidi, et al. (1986) Primary malignant fibrous histiocytoma of the right ventricle and main pulmonary trunk with a review of the literature. Tex Heart Inst J 16: 296-303. [Crossref]

Copyright: $@ 2017$ Newmarch W. This is an open-access article distributed under the terms of the Creative Commons Attribution License, which permits unrestricted use, distribution, and reproduction in any medium, provided the original author and source are credited. 